\title{
Produção de EGP com Diferentes Adesivos PVAc e Sistemas de Prensagem
}

\author{
Cintya Endo ${ }^{1}$, Rosilani Trianoski ${ }^{1}$, Setsuo Iwakiri ${ }^{1}$ \\ ${ }^{1}$ Departamento de Engenharia e Tecnologia Florestal - DETF, Universidade Federal do Paraná - UFPR, \\ Curitiba/PR, Brasil
}

\begin{abstract}
RESUMO
Um dos produtos reconstituídos de grande importância, caracterizado como produto de alto valor agregado, é o painel colado lateralmente, cuja qualidade depende de fatores inerentes a madeira e ao processo produtivo. Neste contexto, o objetivo desta pesquisa é avaliar à qualidade de colagem de painéis EGP de Pinus taeda produzidos com diferentes formulações do adesivo PVAc e sistemas de prensagem. Foi utilizado um delineamento inteiramente casualizado em arranjo fatorial $(4 \times 2)$, respectivamente para formulações do adesivo PVAc (classe D3) e sistemas de prensagem a frio e alta frequência. A qualidade de colagem foi avaliada por meio das normas EN 204:2001 e EN 205:2002. Os resultados não apresentaram diferença estatística significativa a partir da interação entre formulações do adesivo e sistema de prensagem, e nenhum dos tratamentos atingiu o requisito mínimo da norma EN 204:2001, no entanto, os resultados de resistência ao cisalhamento são compatíveis com resultados industriais.
\end{abstract}

Palavras-chave: painel colado lateralmente, EGP, poliacetato de vinila, prensa fria, alta frequência.

\section{Production of EGP with Different PVAc Adhesives and Pressing Systems}

\begin{abstract}
One of the reconstituted products of great importance, characterized as a high-value product, is edge glued panel, whose quality depends on factors inherent to the wood and the production process. In this context, the objective of this research is to evaluate the quality of bonding of EGP panels of Pinus taeda, produced with different PVAc adhesive formulations and pressing systems. A completely randomized design in a factorial arrangement was used $(4 \times 2)$ respectively for PVAc adhesive formulations (class D3) and cold pressing and high frequency. The quality of bonding was evaluated by the standards EN 204: 2001 and EN 205: 2002. The results showed no statistically significant difference from the interaction of adhesive formulations and pressing system, and none of the treatments reached the minimum requirement of EN 204:2001, however, the shear strength results are consistent with industrial results.
\end{abstract}

Keywords: edge glued panel, EGP, polyvinyl acetate, cold press, high frequency. 


\section{INTRODUÇÃO}

A utilização da tecnologia de colagem da madeira possibilita de forma direta a conservação de recursos florestais através do seu aproveitamento integral, unindo peças de diversos tamanhos e formas, gerando produtos reconstituídos (Iwakiri, 2005). Segundo o Laboratório de Produtos Florestais - FPL (USDA Forest Service, 1999), a ligação adesiva tornou-se essencial no desenvolvimento e no crescimento da indústria de produtos reconstituídos e foi um fator-chave na utilização eficiente dos recursos florestais.

Um dos produtos reconstituídos de grande importância, caracterizado como um produto de alto valor agregado (PMVA), é o Edge Glued Panel (EGP), ou painel colado lateralmente, gerado a partir da colagem lateral de sarrafos, podendo estes estar ou não unidos pelo topo com emendas do tipo finger joint (Lopes, 2008). No Brasil, a madeira mais empregada para a geração deste produto é proveniente de florestas plantadas, principalmente de florestas de Pinus (ABIMCI, 2009). Segundo Vick (1999), as espécies de Pinus, principalmente o Pinus taeda, são classificadas como espécies de colagem satisfatória, por possuírem características de massa específica e propriedades químicas adequadas a esta finalidade.

Outro fator extra de importância a ser considerado na colagem de madeiras é o adesivo, para cuja produção de painéis de colagem lateral os adesivos à base de poliacetato de vinila (PVAc) são os mais utilizados (Lopes et al., 2013). De acordo com Bandel (1991), os adesivos PVAc's são produzidos em duas etapas: a primeira consiste na reação de oxidação entre etileno e ácido acético para produzir o acetato de vinil, produto líquido transparente e muito inflamável; e na segunda o acetato de vinil é polimerizado em uma emulsão aquosa, formando uma dispersão aquosa de poliacetato de vinila.

Segundo Pizzi (1983), diferentes componentes são incorporados em um adesivo à base de poliacetato de vinila para a madeira, entre eles a emulsão PVAc (polímero base), álcool polivinílico, plastificantes, solventes, cargas, antiespumantes, pigmentos, biocidas entre outros aditivos, em que cada um tem uma função específica ou gera características desejáveis ao produto final. Estes componentes em diferentes percentuais permitem a produção de uma ampla variedade de adesivos
PVAc's, os quais seguem critérios de classificação de resistência baseados em normas, sendo a norma EN 204:2001 (EN, 2001b) uma das mais utilizadas para essa finalidade, e que classifica os adesivos em grupos de durabilidade distintos, que variam de D1 a D4.

De acordo com a EN 204:2001 (EN, 2001b), a classe D1 compreende os adesivos de uso interior, ou seja, em ambientes onde a umidade de equilíbrio não excede 15\%; a classe D2 contempla adesivos de uso interior com exposições rápidas e esporádicas à umidade, porém a umidade de equilíbrio não deve ser maior que $18 \%$; a classe D3 engloba os adesivos de uso interior com exposições frequentes, porém rápidas à umidade e exterior sem exposição direta ao tempo; e na classe D4 encontram-se os adesivos de uso interior com exposições frequentes e de longa duração à umidade e de uso exterior com proteção adequada.

Esta grande variedade, com amplo espectro de utilização, torna este tipo de adesivo muito difundido na colagem de madeiras e amplamente aceito na indústria (Pizzi, 1983), onde as principais aplicações são a colagem de painéis sarrafeados, emendas "finger joint", laminação e na montagem dos componentes dos móveis (Haubrich et al., 2007). As vantagens são praticidade de uso, formação de união flexível, pouco abrasivo, transparente, fáceis de limpar e permitem um elevado tempo de armazenagem (Heinrich, 2001), baixo custo, segurança no manuseio, inodoro, não inflamável, alta estabilidade (Iwakiri, 2005) e possuem flexibilidade de cura, pois curam a frio ou a quente, onde se incluem as prensas de alta frequência (Conner, 2001; Iwakiri, 2005).

A prensagem a frio é um método pouco automatizado, normalmente realizado em prensas tipo carrossel, que apresenta como vantagens a facilidade operacional e o menor custo tanto de aquisição quanto de manutenção. A principal desvantagem é o tempo de prensagem, que é muito maior quando comparado com o tempo de prensagem a quente ( $30 \mathrm{a} 60$ minutos), gerando dessa forma menor produtividade (Lopes, 2008).

A prensagem a quente pode ocorrer em prensas convencionais ou em prensas de alta frequência, as quais diferem entre si pela forma de transferência de calor. Nas prensas convencionais ocorre o aquecimento e transferência de calor da superfície da madeira para seu interior, em que o aquecimento da madeira pode gerar alterações dimensionais e tensões na linha de cola. Já na 
prensagem em alta frequência, o aquecimento ocorre por radiação eletromagnética, que é um aquecimento volumétrico no qual o campo magnético interage com o material como um todo e o calor é gerado no interior da peça a ser colada, ou mais especificamente, na linha de cola. É um processo rápido quando comparado com a prensagem convencional, e eficiente, devido ao aquecimento ser seletivo na linha de cola, evitando o aquecimento da madeira e as implicações decorrentes das variações dimensionais (Pereira et al., 2005). Embora permita maior produtividade, é um método com maiores custos, tanto de produção quanto de manutenção (Lopes, 2008).

Considerando a importância de desenvolvimentos de estudos relacionados à qualidade de colagem de madeira sólida, especialmente em painéis EGP, à ampla variedade de adesivos à base de poliacetato de vinila e seu desempenho frente às diversas aplicações, assim como os diferentes tipos de prensagem, este trabalho teve como objetivo avaliar o desempenho de diferentes formulações de adesivo PVAc em sistemas de prensagem a frio e alta frequência.

\section{MATERIAL E MÉTODOS}

Foi utilizada nesta pesquisa madeira de Pinus taeda, obtida a partir de lotes comerciais. A adoção desta espécie se deve ao fato de ser a mais utilizada industrialmente para essa finalidade.

As peças com dimensões iniciais de $1.000 \times 95 \times 40 \mathrm{~mm}$ foram desdobradas obtendo-se sarrafos com dimensões de $600 \times 22 \times 20 \mathrm{~mm}$ (comprimento, largura e espessura, respectivamente), os quais posteriormente foram selecionados e submetidos à climatização $\left(20 \pm 2{ }^{\circ} \mathrm{Ce}\right.$ $65 \pm 5 \%$ UR) para obtenção da umidade de equilíbrio.
Após a estabilização foi determinada a massa específica aparente das peças de acordo com a norma Copant 461/1972. O teor de umidade foi obtido de acordo com a norma Copant 460/1972 a partir de peças amostradas no lote de sarrafos, as quais não foram utilizadas para produção dos painéis, mas representavam toda a variabilidade da madeira (COPANT, 1972a, b).

Antes da colagem dos painéis foi realizada uma pré-montagem objetivando colar sarrafos similares entre os tratamentos e, assim, eliminar a influência das características da madeira. Na pré-montagem foram observadas as características de massa específica, percentual de lenho tardio e orientação dos anéis de crescimento, repetindo-se sarrafos com as mesmas características em cada tratamento.

Para a colagem dos painéis foram utilizadas 4 formulações do adesivo polivinil acetato (PVAc), todas produzidas pela empresa Henkel Ltda. e classificadas segundo a norma EN 204 (EN, 2001b) como D3. A avaliação das propriedades dos adesivos foi realizada segundo as normas EN 827:2006, EN 19.092:2000; EN 12.145:2001 e EN 542:2003, obtendo-se respectivamente o teor de sólidos, viscosidade, $\mathrm{pH}$ e densidade (EN, 2000a, 2001a, 2003, 2006).

A gramatura utilizada foi $200 \mathrm{~g} / \mathrm{m}^{2}$, pressão lateral de 0,69 $\mathrm{MPa}\left(7 \mathrm{kgf} / \mathrm{cm}^{2}\right)$, e o tempo de prensagem foi estabelecido de acordo com as especificações indicadas no boletim técnico dos adesivos. Foram produzidos 3 painéis por tratamento, com 5 linhas de cola lateral cada, gerando painéis com dimensões finais de $600 \times 110 \times 20 \mathrm{~mm}$, conforme delineamento experimental apresentado na Tabela 1.

Após a prensagem e estabilização, os painéis foram seccionados para a obtenção dos corpos de prova e

Tabela 1. Delineamento experimental.

Table 1. Experimental design.

\begin{tabular}{cccc} 
Tratamento & Adesivo & $\begin{array}{c}\text { Tempo de prensagem } \\
(\mathbf{m i n})\end{array}$ & $\begin{array}{c}\text { Sistema de Prensagem } \\
1\end{array}$ \\
\hline 2 & PVAc1 & 45 & Prensa fria \\
\hline 3 & PVAc2 & 45 & Prensa fria \\
4 & PVAc3 & 45 & Prensa fria \\
\hline 5 & PVAc4 & 45 & Alta frequência \\
\hline 6 & PVAc1 & 3 & Alta frequência \\
\hline 7 & PVAc2 & 3 & Alta frequência \\
\hline 8 & PVAc3 & 3 & Alta frequência \\
\hline
\end{tabular}


avaliação da qualidade de colagem, os quais seguiram os procedimentos das normas EN 204:2001 e EN 205:2002. Os pré-tratamentos aplicados para esta classe de adesivo conforme a norma EN 204:2001 foram: 1) 7 dias de climatização; 2) 7 dias de climatização + 4 dias em imersão em água; 3 ) 7 dias de climatização + 4 dias em imersão em água +7 dias de climatização (EN, 2001b, 2002).

Após a realização do ensaio de resistência ao cisalhamento da linha de cola, foi realizada a avaliação visual da qualidade de colagem por meio da quantificação do percentual de falha na madeira. A falha na madeira foi classificada como um percentual variando de $0 \%$ a $100 \%$, em que: $0 \%$ representou uma região de cisalhamento na linha de cola sem nenhum vestígio de fibra de madeira nas faces rompidas do corpo de prova, e $100 \%$ representou uma região de cisalhamento na linha de cola integral da madeira, ou seja, observou-se apenas a presença da madeira nas faces rompidas.

O delineamento estatístico utilizado foi o inteiramente casualizado com arranjo fatorial $(4 \times 2)$, cujos fatores foram: formulações de adesivo PVAc e tipos de prensagem, respectivamente, totalizando 8 tratamentos. Os resultados foram submetidos aos testes de Grubbs para avaliar a ocorrência de outliers, Bartlett para verificar a homogeneidade de variâncias e Análise de variância em arranjo fatorial. Quando a Anova indicou a ocorrência de diferença estatística significativa em algum dos parâmetros avaliados e/ou na interação de parâmetros, procedeu-se ao teste de comparação de médias de Tukey. Todos os testes foram efetuados a partir do pacote estatístico Statgraphics Centurion XVI, a 5\% de significância.

\section{RESULTADOS E DISCUSSÕES}

\subsection{Avaliação das propriedades físicas da madeira}

A madeira de Pinus taeda utilizada neste estudo apresentou massa específica aparente média de $0,536 \mathrm{~g} / \mathrm{cm}^{3}$, com coeficiente de variação de $16,89 \%$. A variabilidade da propriedade pode ser justificada por diversos fatores observados no lote de peças, tais como presença de madeira juvenil e adulta, e diferentes percentuais de lenho inicial e tardio.
O teor de umidade médio nas peças foi de 14,30\%, com amplitude de $13,72 \%$ a $14,80 \%$, em que este valor é superior ao valor especificado pelo fabricante para a colagem, de $8 \%$ a $12 \%$. Por outro lado, os valores encontrados são similares aos mencionados por Tienne (2006), o qual relata que a faixa de umidade que confere melhor adesão para madeira situa-se na faixa de 6\% a 14\%. Segundo Prata (2010), que também desenvolveu colagens com teores de umidade superiores aos recomendados pelo fabricante (TU: 14,56\% e CV: $2,94 \%)$, apesar de os valores médios estarem acima do valor recomendado, baixos coeficientes de variação indicam que todas as peças são coladas na mesma faixa de umidade, descartando-se a hipótese de alguma peça ter um comportamento diferenciado durante o processo de colagem em função da influência deste fator.

\subsection{Avaliação das propriedades físicas e químicas dos adesivos}

Na Tabela 2 são apresentados os resultados médios das propriedades físicas e químicas dos diferentes tipos de adesivos.

A partir dos resultados apresentados na Tabela 2, verifica-se que os valores das propriedades determinadas em laboratório estão, na maioria dos casos, de acordo com os valores especificados pelo fabricante.

Para a propriedade teor de sólidos, nota-se que os adesivos PVAc1 e PVAc2 apresentaram percentual de sólidos superior ao especificado pelo fabricante, o que indica ser benéfico à colagem, pois existem mais componentes formando ligações adesivas ao substrato, assim como maior será a tensão necessária para promover o descolamento, no entanto, são adesivos de maior custo. Ainda segundo Bandel (1991), a quantidade de sólidos presentes na resina está diretamente relacionada com a velocidade de formação da película adesiva, ou seja, quanto maior for a quantidade de água (menor o teor de sólidos) na resina, mais lenta será a velocidade de formação da película devido ao maior tempo de evaporação desta água. Assim, pressupõem-se que os adesivos 3 e 4, além da menor resistência, poderão apresentar maior tempo de cura do adesivo, implicando diretamente aumento do tempo de prensagem, assim como menor produtividade. Por outro lado, possivelmente devem ser adesivos de menor custo, apesar de estarem dentro do valor especificado pelo fabricante. 
Tabela 2. Propriedades físicas e químicas dos adesivos.

Table 2. Physical and chemical properties of adhesives.

\begin{tabular}{|c|c|c|c|}
\hline Propriedade & Adesivo & Valor médio $^{1}$ & Fabricante $^{2}$ \\
\hline \multirow{4}{*}{ Teor de sólidos (\%) } & PVAc 1 & $47,61 \%$ & $45 \%$ \\
\hline & PVAc 2 & $51,06 \%$ & $47 \%$ \\
\hline & PVAc 3 & $42,59 \%$ & $42-47 \%$ \\
\hline & PVAc 4 & $43,75 \%$ & $40-46 \%$ \\
\hline \multirow{4}{*}{ Viscosidade (cPs) } & PVAc 1 & 3.758 & 4.000 \\
\hline & PVAc 2 & 4.067 & 4.000 \\
\hline & PVAc 3 & 2.062 & $3.500-5.000$ \\
\hline & PVAc 4 & 3.416 & $2.500-3.600$ \\
\hline \multirow{4}{*}{$\mathrm{pH}$} & PVAc 1 & 3,86 & 4,0 \\
\hline & PVAc 2 & 3,36 & 4,0 \\
\hline & PVAc 3 & 4,33 & $3,5-4,5$ \\
\hline & PVAc 4 & 3,69 & $3,0-4,0$ \\
\hline \multirow{4}{*}{ Densidade $\left(\mathrm{g} / \mathrm{cm}^{3}\right)$} & PVAc 1 & 1,09 & $1,05-1,10$ \\
\hline & PVAc 2 & 1,10 & $1,05-1,10$ \\
\hline & PVAc 3 & 1,07 & $1,05-1,10$ \\
\hline & PVAc 4 & 1,07 & $1,02-1,07$ \\
\hline
\end{tabular}

${ }^{1}$ : Valor obtido em laboratório; ${ }^{2}$ : Valor especificado pelo fabricante.

Em relação à viscosidade, verifica-se que o adesivo PVAc 3 apresentou valor médio bastante inferior ao informado pelo boletim técnico do fabricante, podendo gerar implicação na penetração do adesivo na madeira e na qualidade de colagem. De acordo com Iwakiri (2005), quanto menor a viscosidade, maior a penetração e absorção do adesivo pela madeira, formando uma linha de cola faminta, assim como uma colagem de baixo desempenho. Por outro lado, se a viscosidade for elevada, menor é a penetração do adesivo na estrutura capilar da madeira, formando linha de cola espessa, ocasionando insuficiência na interligação do sistema madeira-adesivo-madeira, e também uma baixa qualidade de colagem.

Outro fato importante a ser destacado é que algumas vezes a baixa viscosidade do adesivo está associada a uma maior quantidade de água na sua composição e consequente menor teor de sólidos. Logo, esta pode ser uma das explicações para o adesivo PVAc 3 , já que ele apresentou o menor teor de sólidos (42,59\%) entre os adesivos avaliados.

Em relação ao $\mathrm{pH}$, todos os adesivos mostraram valores próximos ou de acordo com a faixa de variação apresentada pelo fabricante, e com a literatura, que relata valores de $\mathrm{pH}$ de 4,0 a 5,0 para o PVA (Marra, 1992). $\mathrm{O}$ adesivo PVAc 2 mostrou-se o mais ácido, e embora esta ligeira acidez possa não prejudicar diretamente a qualidade de colagem, pode provocar manchas e descolorações quando em contato prolongado com ferro (Marra, 1992). Ainda segundo este autor, quando o pH do adesivo é inferior a 3, existe a possibilidade de degradação ácida da madeira, sendo necessário a adição de soluções-tampão para proteger o pH. Em complemento, Iwakiri (2005) relata que adesivos com $\mathrm{pH}$ muito baixo podem provocar formação excessiva de espuma na mistura, prejudicando sensivelmente a aplicação. Vale ressaltar ainda que o pH determina a velocidade, o grau das reações e as configurações das moléculas do adesivo, assim como sua validade (Marra, 1992).

Quanto à densidade, todos os adesivos apresentaram valores no intervalo especificado pelo fabricante, não sendo constatadas discrepâncias, assim como possíveis influências sobre a qualidade de colagem.

\subsection{Avaliação da resistência da linha de cola}

Na Tabela 3 são apresentados os valores da Anova em arranjo fatorial e os resultados médios para o efeito principal adesivo.

Avaliando o fator adesivo, nota-se que os corpos de prova obtidos a partir dos painéis produzidos com o adesivo PVAc2 apresentaram, na maioria dos pré-tratamentos, os maiores valores médios numéricos de resistência, podendo este resultado estar associado ao maior teor de sólidos, conforme apresentado na 
Tabela 3. Resultados médios do efeito do adesivo sobre a resistência da linha de cola.

Table 3. Average results of the effect of adhesive on the glue line resistance.

\begin{tabular}{|c|c|c|c|}
\hline \multicolumn{4}{|c|}{ Resistência da linha de cola ao cisalhamento (MPa) } \\
\hline Adesivo & Seco $12 \%^{1}$ & Imersão $^{2}$ & Imersão + climatização ${ }^{3}$ \\
\hline$A_{1}-$ PVAc 1 & $\begin{array}{l}5,46^{60} \\
(25,14)\end{array}$ & $\begin{array}{c}1,47^{0} \\
(58,22)\end{array}$ & $\begin{array}{c}5,36^{37} \mathrm{a} \\
(29,39)\end{array}$ \\
\hline $\mathrm{A}_{2}-\mathrm{PVAc} 2$ & $\begin{array}{c}5,55^{85} \\
(19,76)\end{array}$ & $\begin{array}{c}1,33^{0} \\
(65,45)\end{array}$ & $\begin{array}{c}5,74^{42} \mathrm{a} \\
(41,89)\end{array}$ \\
\hline $\mathrm{A}_{3}-\mathrm{PVAc} 3$ & $\begin{array}{l}5,35^{77} \\
(25,25)\end{array}$ & $\begin{array}{c}1,29^{0} \\
(84,18)\end{array}$ & $\begin{array}{c}5,02^{29} \mathrm{ab} \\
(41,31)\end{array}$ \\
\hline $\mathrm{A}_{4}-\mathrm{PVAc} 4$ & $\begin{array}{l}5,12^{64} \\
(25,51)\end{array}$ & $\begin{array}{c}0,74^{0} \\
(60,18)\end{array}$ & $\begin{array}{c}3,36{ }^{20} b \\
(59,56)\end{array}$ \\
\hline $\mathrm{F}$ & 0,38 & 2,92 & 5,23 \\
\hline$p$ & $0,7649^{\text {ns }}$ & $0,0507^{\mathrm{ns}}$ & $0,0027^{\star}$ \\
\hline
\end{tabular}

${ }^{1}: 7$ dias de climatização a $12 \%$; $2: 7$ dias de climatização a $12 \%+4$ dias de imersão em água; ${ }^{3}: 7$ dias de climatização a $12 \%+4$ dias de imersão em água +7 dias de climatização a $12 \%$; ${ }^{*}$ significativo ao nível de $95 \%$ de probabilidade; ns $=$ não significativo ao nível de 95\% de probabilidade; valores entre parênteses indicam o coeficiente de variação das amostras em \%; valores sobrescritos indicam a falha na madeira em \%.

Tabela 2. Normalmente, maior teor de sólidos no adesivo implica benefícios de qualidade de colagem, pois existem mais componentes formando ligações adesivas ao substrato, sendo necessária maior tensão para promover o descolamento ou ruptura da peça colada. Além da resistência ao cisalhamento, verifica-se que o teor de sólidos exerceu ligeira influência sobre o percentual de falha da madeira, onde as peças coladas com o adesivo com maior teor de sólidos apresentaram os maiores valores médios.

Foi constatada diferença estatística significativa apenas no pré-tratamento de imersão + climatização, onde os adesivos PVAc 1, PVAc 2 e PVAc 3 apresentaram-se estatisticamente iguais, formando um grupo homogêneo entre si. Por outro lado, o adesivo PVAc 3 não diferiu estatisticamente do adesivo PVAc 4.

A Tabela 4 mostra o efeito do sistema de prensagem sobre os resultados médios da resistência da linha de cola ao cisalhamento.

Em relação ao sistema de prensagem, não foi constatada diferença estatística significativa para o ensaio seco, e os corpos de prova avaliados após imersão, e, em imersão + climatização, não apresentaram um efeito claro desta variável. De maneira geral, os resultados obtidos para os diferentes sistemas de prensagem são muito parecidos entre si, o que permite afirmar que esta variável não gera grandes diferenças na resistência da colagem lateral.

Na Tabela 5 são apresentados os resultados médios das interações entre os diferentes adesivos e sistemas de prensagem sobre a resistência da linha de cola.
$\mathrm{Na}$ análise da interação entre adesivos e sistemas de prensagem, verifica-se que em nenhum dos pré-tratamentos houve diferença estatística significativa, indicando que, nas condições de realização deste trabalho, não há necessidade de distinção de adesivos e tipos de prensas, permitindo dessa forma minimização dos custos e maximização da produtividade, o que se torna uma vantagem industrial. Numa abordagem mais sistêmica, observa-se que os painéis colados com o adesivo PVAc 2 também tenderam a apresentar os maiores resultados de resistência ao cisalhamento, assim como de falha na madeira, indicando a influência positiva do teor de sólidos.

Outro fato claramente constatado foi a perda de resistência em função do pré-tratamento de imersão em água, o qual é explicado pela característica termoplástica do adesivo, ou seja, deste se tornar reversível na presença de água, onde as forças de coesão que unem as moléculas do adesivo e o substrato ficam reduzidas, resultando numa linha de cola de baixa resistência. Por outro lado, o pré-tratamento de climatização inicial por 7 dias, imersão e posterior climatização por mais 7 dias, não proporcionou perdas acentuadas de resistência. Este resultado também pode ser justificado pelas características termoplásticas do adesivo, em que neste pré-tratamento possivelmente houve "reversibilidade parcial" da cura durante o processo de imersão, mas com a posterior climatização dos corpos de prova, o adesivo retomou sua capacidade adesiva. Este comportamento pode ser corroborado pela afirmação de Bandel (1991), que após estudos afirma que o adesivo PVAc tem a 
Tabela 4. Resultados médios do efeito do sistema de prensagem sobre a resistência da linha de cola.

Table 4. Average results of the effect of pressing system on the glue line resistance.

\begin{tabular}{|c|c|c|c|}
\hline \multicolumn{4}{|c|}{ Resistência da linha de cola ao cisalhamento (MPa) } \\
\hline Sistema de prensagem & Seco $12 \%^{1}$ & Imersão $^{2}$ & Imersão + climatização ${ }^{3}$ \\
\hline S1 - Prensa fria & $\begin{array}{l}5,39^{78} \\
(22,41)\end{array}$ & $\begin{array}{c}0,94^{0} \mathbf{b} \\
(66,04)\end{array}$ & $\begin{array}{c}4,96^{30} \mathbf{a} \\
(40,17)\end{array}$ \\
\hline$S_{2}-$ Alta frequência & $\begin{array}{l}5,35^{65} \\
(21,10)\end{array}$ & $\begin{array}{l}1,49^{0} \mathbf{a} \\
(68,95)\end{array}$ & $\begin{array}{c}4,46^{29} \mathbf{b} \\
(45,36)\end{array}$ \\
\hline $\mathrm{F}$ & 0,02 & 7,22 & 5,15 \\
\hline$p$ & $0,8766^{\mathrm{ns}}$ & $0,0091^{*}$ & $0,0265^{*}$ \\
\hline
\end{tabular}

1: 7 dias de climatização a $12 \%$; ${ }^{2}: 7$ dias de climatização a $12 \%+4$ dias de imersão em água; ${ }^{3}: 7$ dias de climatização a $12 \%+4$ dias de imersão em água +7 dias de climatização a $12 \%$; ${ }^{*}$ significativo ao nível de $95 \%$ de probabilidade; ${ }^{\text {ns }}=$ não significativo ao nível de 95\% de probabilidade; valores entre parênteses indicam o coeficiente de variação das amostras em \%; valores sobrescritos indicam a falha na madeira em \%.

Tabela 5. Resultados médios das interações entre os fatores adesivos e sistemas de prensagem.

Table 5. Average results from interactions between the factors adhesive and pressing systems.

\begin{tabular}{|c|c|c|c|}
\hline \multicolumn{4}{|c|}{ Resistência da linha de cola ao cisalhamento (MPa) } \\
\hline Adesivo & Seco $12 \%^{1}$ & Imersão $^{2}$ & Imersão + climatização $^{3}$ \\
\hline 1 - (PVAc 1 - PF) & $\begin{array}{l}5,50^{59} \\
(29,62)\end{array}$ & $\begin{array}{c}1,16^{0} \\
(62,92)\end{array}$ & $\begin{array}{l}5,51^{19} \\
(21,77)\end{array}$ \\
\hline 2 - (PVAc 2 - PF) & $\begin{array}{c}5,63^{87} \\
(10,97)\end{array}$ & $\begin{array}{c}1,02^{0} \\
(75,18)\end{array}$ & $\begin{array}{c}7,17^{49} \\
(28,48)\end{array}$ \\
\hline 3 - (PVAc 3 - PF) & $\begin{array}{l}5,41^{83} \\
(24,60)\end{array}$ & $\begin{array}{c}0,92^{0} \\
(62,28)\end{array}$ & $\begin{array}{l}5,55^{31} \\
(18,24)\end{array}$ \\
\hline 4 - (PVAc 4 - PF) & $\begin{array}{l}5,03^{83} \\
(22,43)\end{array}$ & $\begin{array}{c}0,66^{0} \\
(53,49)\end{array}$ & $\begin{array}{l}3,28^{19} \\
(62,72)\end{array}$ \\
\hline 5 - (PVAc 1 - AF) & $\begin{array}{l}5,42^{61} \\
(22,79)\end{array}$ & $\begin{array}{c}1,78^{0} \\
(54,21)\end{array}$ & $\begin{array}{l}5,22^{54} \\
(34,00)\end{array}$ \\
\hline 6 - (PVAc $2-\mathrm{AF})$ & $\begin{array}{l}5,46^{83} \\
(25,61)\end{array}$ & $\begin{array}{c}1,26^{0} \\
(61,50)\end{array}$ & $\begin{array}{l}4,32^{15} \\
(39,79)\end{array}$ \\
\hline 7 - (PVAc 3 - AF) & $\begin{array}{l}5,30^{70} \\
(23,27)\end{array}$ & $\begin{array}{c}2,14^{0} \\
(71,57)\end{array}$ & $\begin{array}{c}4,48^{26} \\
(58,94)\end{array}$ \\
\hline 8 - (PVAc 4 - AF) & $\begin{array}{c}5,20^{44} \\
(26,97)\end{array}$ & $\begin{array}{c}0,80^{0} \\
(69,95)\end{array}$ & $\begin{array}{l}3,44^{21} \\
(60,23)\end{array}$ \\
\hline $\mathrm{F}$ & 0,06 & 1,36 & 2,24 \\
\hline$p$ & $0,9802^{\text {ns }}$ & $0,2638^{\text {ns }}$ & $0,0918^{\mathrm{ns}}$ \\
\hline
\end{tabular}

${ }^{1}: 7$ dias de climatização a $12 \%{ }^{2}: 7$ dias de climatização a $12 \%+4$ dias de imersão em água; ${ }^{3}: 7$ dias de climatização a $12 \%+4$ dias de imersão em água +7 dias de climatização a $12 \%$; ${ }^{\text {ns }}=$ não significativo ao nível de $95 \%$ de probabilidade; valores entre parênteses indicam o coeficiente de variação das amostras em \%; valores sobrescritos indicam a falha na madeira em \%.

capacidade de retornar à sua forma coesiva quando exposto à umidade em um tempo curto de exposição.

Ainda em observação deste pré-tratamento, nota-se que o sistema de prensagem a frio proporcionou um ligeiro aumento na resistência ao cisalhamento em 3 dos 4 adesivos utilizados, quando estes são comparados com o ensaio em estado seco ou climatizado, onde o maior incremento foi apresentado pelo adesivo PVAc 2, o qual possui maior teor de sólidos.

Avaliando os valores de falha na madeira, percebe-se um alto percentual de falha no pré-tratamento a seco para todos os adesivos testados, tanto na prensagem a frio quanto na prensagem em alta frequência, significando que a linha de cola foi mais resistente que a madeira. Já no pré-tratamento de imersão, o percentual de falha foi de $0 \%$, demonstrando que os adesivos possuem grande vulnerabilidade quando expostos à umidade por um longo período de tempo. No pré-tratamento de imersão + climatização, percebe-se que os corpos de prova voltaram a apresentar falha na madeira, o que demonstra a capacidade de o adesivo retornar à sua capacidade adesiva, mesmo após o período de exposição à umidade. 
Considerando os requisitos mínimos da norma EN 204:2001 para a classe D3, à qual pertencem os adesivos avaliados, verifica-se que nenhum dos adesivos/tratamentos apresentou o valor mínimo de $10 \mathrm{MPa}$ no ensaio seco; apenas o tratamento 7, produzido com o adesivo PVAc 3 e prensagem em alta frequência, atingiu o requisito mínimo de $2 \mathrm{MPa}$ no pré-tratamento de imersão; nenhum tratamento atingiu a resistência mínima de $8 \mathrm{MPa}$ no pré-tratamento de imersão + climatização, sendo, portanto, classificados como não conformes perante esta norma. Por outro lado, deve-se considerar que como esta norma avalia a qualidade do adesivo, ela sugere como substrato a madeira de Fagus sylvatica, que apresenta propriedades anatômicas, químicas, físicas e mecânicas distintas da madeira de Pinus taeda, podendo gerar diferenças no processo de colagem e seu posterior comportamento, devendo-se, neste caso, observar as exigências do mercado interno.

Em função dos valores obtidos, estes são comparados com os resultados de Lopes (2008), que ao avaliar diferentes adesivos a partir de colagens em laboratório e colagens industriais, encontrou para amostras coladas com adesivo PVAc em laboratório, resistência ao cisalhamento de 7,21 a 9,10 $\mathrm{MPa}$ a seco, e de 1,54 a $1,91 \mathrm{MPa}$ no ensaio de imersão, falhas na madeira de $39 \%$ a $49 \%$ e de $3 \%$ a $6 \%$, e elevados coeficientes de variação, $82 \%$ e $85 \%$, respectivamente para condições mencionadas. Dessa forma, observa-se que embora a resistência ao cisalhamento em ambas as condições de umidade sejam ligeiramente superiores ao desta pesquisa, também não atingem o valor mínimo preconizado pela norma EN 204:2001. Já a falha na madeira no ensaio a seco do presente trabalho foi superior, e o coeficiente de variação foi menor, indicando maior homogeneidade nos resultados.

Em comparação com os resultados encontrados a partir das amostras coletadas na indústria, verifica-se que a resistência ao cisalhamento foi de 5,50 $\mathrm{MPa}$ no ensaio seco e de 2,41 MPa no ensaio de imersão, falhas de $83 \%$ e $28 \%$, respectivamente, e coeficiente de variação de $30 \%$, indicando que no ensaio seco os resultados deste trabalho são similares ao que é obtido industrialmente, ligeiramente inferiores na condição úmida e mais homogêneos, onde esta última característica indica menor variabilidade em peças industriais, o que possivelmente é explicado por fatores inerentes ao próprio processo produtivo.

\section{CONCLUSÕES}

- A madeira de Pinus taeda apresentou teor de umidade ligeiramente superior ao recomendado pela fabricante para colagens com adesivo PVAc;

- Os adesivos apresentaram propriedades físicas e químicas de acordo com os valores especificados no boletim técnico do fabricante, ou próximos destes;

- Os adesivos PVAC 1 e PVAc 2 apresentaram teor de sólidos superior ao indicado pelo fabricante;

- Não foram observadas grandes diferenças de resistências ao cisalhamento a partir das diferentes formulações do adesivo PVAc;

- Não houve uma influência clara da variável sistema de prensagem;

- Não foi constatada diferença estatística significativa a partir da interação entre formulações do adesivo e sistema de prensagem;

- Nenhum dos tratamentos atingiu o requisito mínimo da norma EN 204:2001;

- Apesar de os resultados não atingirem o requisito mínimo preconizado pela norma $\mathrm{EN}$ 204:2001, são compatíveis com resultados obtidos industrialmente.

\section{AGRADECIMENTOS}

À empresa Henkel Ltda pela doação dos adesivos e concessão de uso da prensa de alta frequencia.

\section{STATUS DA SUBMISSÃO}

Recebido: 16 maio, 2015

Aceito: 7 set., 2016

\section{AUTOR(ES) PARA CORRESPONDÊNCIA}

\section{Rosilani Trianoski}

Departamento de Engenharia e Tecnologia Florestal - DETF, Universidade Federal do Paraná - UFPR, Av. Pref. Lothário Meissner, 632, Campus III da UFPR, Jardim Botânico, CEP 80210-170, Curitiba, PR, Brasil e-mail: rosilani@ufpr

\section{REFERÊNCIAS}

Associação Brasileira da Indústria de Madeira Processada Mecanicamente - ABIMCI. Estudo setorial 2009: indústria de madeira processada mecanicamente: ano base 2008. Curitiba; 2009. 48 p. 
Bandel A. Adhesivos y tecnología del encolado en la industria de la madera. Milano: Rib\&s; 1991.

Comissão Panamericana de Normas Técnicas - COPANT. COPANT 460: método de determinación de la humidad. La Paz; 1972a.

Comissão Panamericana de Normas Técnicas - COPANT. COPANT 461: maderas: determinación del peso especifico aparente. La Paz; 1972b.

Conner AH. Wood: adhesives. In: Buschow KHJ, editor. Encyclopedia of materials: science and technology. USA: Elsevier Science; 2001.

European Standard - EN. EN 19.092: adhesives: determination of viscosity. Bruxelas; 2000a.

European Standard - EN. EN 12.145: adhesives: adhesives of pH: test method. Bruxelas; 2001a.

European Standard - EN. EN 204: classification of thermoplastic wood adhesives for non-structural applications. Bruxelas; $2001 \mathrm{~b}$.

European Standard - EN. EN 205: Adhesives: wood adhesives for non-structural applications: determination of tensile shear stregth of lap joints. Bruxelas; 2002.

European Standard - EN. EN 542: adhesives: determination of density. Bruxelas; 2003.

European Standard - EN. EN 827: adhesives: determination of conventional solids content and mass solids. Bruxelas; 2006.

Haubrich JL, Gonçalves C, Tonet A. Adesivos vinílicos apresentam soluções para madeira. Revista da Madeira 2007; 103: 66-70

Heinrich $\mathrm{H}$. Wood adhesion and glued product. Bruxelas: COST Action E13 WG; 2001.

Iwakiri S. Painéis de madeira reconstituída. Curitiba: Fupef; 2005.
Lopes MC. Espectroscopia no infravermelho próximo aplicada na avaliação de painéis de madeira colados lateralmente [tese]. Curitiba: Universidade Federal do Paraná; 2008.

Lopes MC, Muniz GIB, Matos JLM, Tanobe VOA, Chinasso CAF, Rosso S. Resistência da linha de cola de Painéis de Pinus taeda colados lateralmente com diferentes adesivos. Cerne 2013; 19(4): 613-619. http://dx.doi.org/10.1590/ S0104-77602013000400011.

Marra AA. Technology of wood bonding. New York: Van Nostrand Reinhold; 1992.

Pereira J, Carvalho L, Martins J. Determinação do tempo de prensagem óptimo de placas de madeira maciça por alta frequência. In: Actas do $5^{\circ}$ Congresso Florestal Nacional; 2005; Viseu. Viseu: Instituto Politécnico de Viseu; 2005.

Pizzi A. Wood adhesives: chemistry and technology. New York: Marcell Dekker; 1983.

Prata JG. Estudo da viabilidade tecnológica do uso de espécies de Pinus tropicais para produção de painéis colados lateralmente (Edge Glued Panels - EGP) [tese]. Curitiba: Universidade Federal do Paraná; 2010.

Tienne DLC. Qualidade da adesão de juntas coladas expostas a condições de serviço externo e interno [dissertação]. Seropédica: Instituto de Florestas, Universidade Federal Rural do Rio de Janeiro; 2006.

United State Forest Products Laboratory - USDA Forest Service. Wood handbook: wood as an engineering material. Madison: Department of Agriculture, Forest Service, Forest Products Laboratory; 1999. General Technical Report FPL-GTR-113.

Vick CB. Adhesive bonding of wood materials. In: United State Forest Products Laboratory - USDA Forest Service. Wood handbook: wood as an engineering material. Madison: Department of Agriculture, Forest Service, Forest Products Laboratory; 1999. 